Mitteilungen der Österreichischen Geographischen Gesellschaft, 159. Jg. (Jahresband), Wien 2017, S. 359-364

\title{
300 JAHRE INGENIEUR-AKADEMIE 1717/2017 200 Jahre Franziszeischer Kataster 1817/2017 100 JAHRE VerEINBARUNG VON BUdAPEST 1917/2017
}

\author{
Gerhard L. FASCHING, Wien und Salzburg*
}

\begin{abstract}
Diese drei kulturellen Großtaten auf dem Gebiet des Geoinformationswesens mit gravierenden Auswirkungen auf Österreich und Mitteleuropa bis heute wurden 2017 durch eine Reihe von hochkarätigen militärischen und zivilen Veranstaltungen gewürdigt. Solche Jubiläen bilden einen guten Anlass, aus heutiger Sicht diese Ereignisse quellenkritisch und im wissenschaftlichen Kontext neu zu bewerten. Dabei musste mit Erstaunen festgestellt werden, dass alle drei Ereignisse, trotz ihrer überragenden fachlichen Bedeutung, selbst in Fachkreisen wenig bekannt und noch weniger bewusst sind. Im Laufe der Zeit hat sich wegen unkritischer Übernahme von Aussagen und wegen Animositäten zwischen der militärischen und zivilen Geschichtsschreibung eine Reihe von Unschärfen und Falschbeurteilungen ergeben. Einige dieser neuen Forschungsergebnisse sollen mit dem vorliegenden Bericht thematisiert werden.
\end{abstract}

Die Ingenieur-Akademie Wien zur Ausbildung von Militär- und Zivilingenieuren wurde auf Anregung des Präsidenten des Hofkriegsrates (entspricht einem heutigen Verteidigungsminister) Eugen Prinz von Savoyen-Carigan (1663-1736) durch Kaiser Karl VI. am 24. Dezember 1717 gegründet (FASCHING 2017b). Treibende Kräfte für dieses Projekt waren die beiden geographischen Ingenieure (= Astronomen, Geodäten, Topographen und Kartographen, im heutigen Wissenschaftsverständnis aber keine Geographen) und Direktoren der Ingenieur-Akademie Oberstleutnant Leander Conte Anguissola (1652-1720) und Prof. Dr. Johann Jakob von Marinoni (1676-1755).

Besonders der kaiserliche Hofmathematiker MARINONI erwarb sich große Verdienste als Astronom (u.a. Gründer der heutigen Universitätssternwarte), Geodät (erste exakte Basismessungen für Triangulierungen), Topograph (Schöpfer des Mailänder Katasters mittels von ihm entwickelter moderner Messtischaufnahme, Vorbild für die folgenden europäischen Katastervermessungen und Landesaufnahmen) und als Erzieher (in den naturwissenschaftlichen Fächern) der Erzherzogin und späteren Kaiserin Maria Theresia. Darüber hinaus entwarf er die Befestigungsanlagen des Linienwalles rund um die Vorstädte von Wien und erstellte im Auftrag von Kaiser Joseph I. den ersten exakten Plan von Wien sowie einen Jagdatlas für Kaiser Karl VI. Weiters war er an den Planungen der merkantilistischen Post- und Commerzialstraßen der habsburgischen Kronländer (so an der von Pionieren erbauten ersten Kunststraße über den Semmering) beteiligt. Anlässlich des Gedenkens an 200 Jahre Kataster wurde an seinem Begräbnisort, der Krypta der Schottenkirche Wien, eine Gedenktafel enthüllt (Hiermanseder \& KöNIG 2017). Am 14. November 2017 fand dort eine feierliche Kranzniederlegung von Vertretern des Österreichischen Militärischen Geo-Dienstes, des Bundesamtes für Eich- und Vermessungswesen sowie des früheren und jetzigen Leiters der Kartensammlung des Kriegsarchivs statt.

\footnotetext{
* Prof. Dr. Gerhard L. FAsching, Brigadier i.R., Krottenbachstraße 189, A-1190 Wien; E-Mail: gerhard. fasching@sbg.ac.at
} 


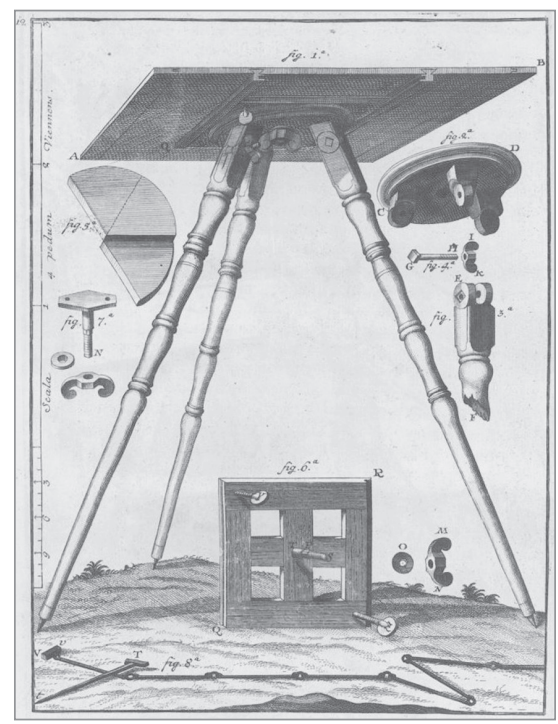

Quelle: De re ichnographica, cuius hodierna praxis exponitur, et propriis exemplis pluribus illustratur, 1751, S. 12, Eidgenössische Technische Hochschule Zürich/Bibliothek

Abb. 1: Von Johann J. MarinONI weiterentwickelter Messtisch Muster 1718 für den Mailänder Kataster und für die Topographische Landesaufnahme. Dadurch konnten Verbesserungen hinsichtlich Genauigkeit und Beschleunigung der Geländearbeit erzielt werden. Im Vordergrund eine Messkette mit einer Länge von 10 Wiener Klaftern $(18,96 \mathrm{~m})$ zur genauen Basismessung bei lokalen Triangulierungen, z.B. beim gemeindeweisen Kataster sowie Lager- und Festungsbau.

Interessant ist es festzuhalten, dass von Schels (1836) und LichtenBERGER (2009) auch eine Ingenieur-Akademie in Brüssel [Bruxelles/Brussel] angeführt wurde. Vermutlich dürfte es sich dabei aber um eine Verwechslung mit der 1713 gegründeten „Brüsseler Academie“ handeln. Diese war aber keine höhere Techniker-Ausbildungseinrichtung wie die Wiener Ingenieur-Akademie, sondern ein interdisziplinärer Gelehrtenzusammenschluss im Sinne des aufgeklärten Absolutismus (FASCHING 2017b).

Weiters musste mit Erstaunen festgestellt werden, dass das Geoinformationswesen als ganz wichtiger Bildungsinhalt der militärischen und militärtechnischen Ausbildung zunächst bei den habsburgischen und dann bei den österreichischen bzw. österreich-ungarischen Land- und Seestreitkräften bis Ende des 19. Jhs. in der damaligen Militärliteratur nur kursorisch behandelt wurde. Besonders augenfällig war dies in den bisher erschienenen Festschriften zur Ingenieur-Akademie und deren Nachfolgeorganisationen (k.k. Ingenieurschule, k.k. Genieakademie, k.k. Polytechnisches Institut, k.k. Technische Hochschule Wien, k.u.k. Technische Militärakademie). Diese Diskrepanz lässt sich aber leicht erklären, wenn man weiß, dass in Mitteleuropa seit dem Dreißigjährigen Krieg die strikte Geheimhaltung von Geoinformationen eine Selbstverständlichkeit war. Hier sei nur auf die Erste österreichische Theresianisch-Josephinische Landesaufnahme 1763-1785 der habsburgischen Kronländer hingewiesen, bei der aus Geheimhaltungsgründen lediglich zwei Exemplare (!) jedes Kartenblattes hergestellt wurden. Eine extreme Geheimhaltung von Geoinformationen erfolgte auch nach den traumatischen Erfahrungen im Großen Vaterländischen Krieg durch die Sowjetunion im Kalten Krieg von den Teilnehmerstaaten des Warschauer Vertrages. 
Ein weiterer Mythos konnte durch Archivarbeit im Kriegsarchiv Wien aufgeklärt werden, nämlich der von der hohen Qualität und Quantität der Landesaufnahmen in Oberitalien und Dalmatien ab 1802 durch das Deposito della guerra (Kriegsdepot) der Zisalpinen Republik bzw. des Königreiches Italien von Napoleons Gnaden. Diese Vermessungsarbeiten und Landesbeschreibungen wurden nicht durch Franzosen, sondern von Absolventen der Ingenieur-Akademie Wien und von italienischen Hilfskräften durchgeführt! Dabei konnte man auf die Tradition und Berufspraxis seit dem Mailänder Kataster 1720-1760 unter MARINONI zurückgreifen.

Auch die sich seit mehr als 150 Jahren haltende hartnäckige Mär vom Fehlen der Ersten Landesaufnahme in Tirol, weil es dort bereits die Anich-Karte gab, konnte entkräftet werden. Der Atlas Tyrolensis 1774 war eine großartige geodätische und kartographische Leistung von Peter ANICH und Blasius HuEBER. Das Kartenwerk war aber für die militärischen Zwecke des Generalquartiermeisterstabes unbrauchbar, weil Wiesen und Wasserstellen zur Versorgung von Mensch und Tier maßstabbedingt nicht ausgewiesen wurden. Wegen anderer Prioritäten am südöstlichen Kriegsschauplatz - gegen die Osmanen - wurde zwar mehrmals mit einer Landesaufnahme in der Gefürsteten Grafschaft Tirol begonnen. Sie wurde aber erst nach den militärischen Erfolgen des republikanischen Konsuls Napoleon in Oberitalien ernsthaft in Angriff genommen. Nach der Niederlage der Österreicher 1809 war eine der ersten Handlungen der Bayern, alle vorhandenen Landesaufnahmen und Landesbeschreibungen von Tirol zu beschlagnahmen. Obwohl die Bayern gemäß Wiener Kongress verpflichtet gewesen wären, diese Geoinformationen zu retournieren, dauerte es bis in die 1830er Jahre, bis die Unterlagen kopiert, ausgewertet und Innsbruck zurückgegeben wurden. Dort ruhen sie bis heute im Tiroler Landesarchiv.

Wurde die Rolle des Militärs für das Geoinformationswesen im 18. Jh. eher unterschätzt (vgl. KRETSChMER et al. 2004), wurde sie im 19. und 20. Jh. eher überschätzt. So konnte zum Beispiel erst durch den für das gesamte Staatsgebiet vorliegenden Franziszeischen Kataster die Dritte österreichisch-ungarische Franzisko-Josephinische Landesaufnahme in der kurzen Zeit 1869-1887/88 in Form von 2.780 mehrfarbigen Aufnahmeblättern („Messtischblättern“ oder „Sectionen“) 1:25.000 bzw. 752 Kartenblättern der Spezialkarte 1:75.000 realisiert werden.

Auf Initiative der Kommission der Österreichischen Akademie der Wissenschaften (ÖAW) für die wissenschaftliche Zusammenarbeit mit Dienststellen des Bundesministeriums für Landesverteidigung wurde das 300-jährige Jubiläum der Gründung der Ingenieurakademie mit einem Festakt am 15. November 2017 in der Sala Terrena der Landesverteidigungsakademie Wien und einer Festschrift für alle Militärtechnikbereiche gewürdigt. Das Geoinformationswesen wurde dabei von zwei Beiträgen (FASChing 1717-2000, GNASER \& TEICHMANN 2000-2017) abgedeckt. Vorgestaffelt fand am 14. November in der Kreuzkapelle des Domes zu Sankt Stephan in Wien am Grabmal von Prinz Eugen eine offizielle Kranzniederlegung des Bundesheeres und der ÖAW mit Gardesoldaten und einem Hornisten der Gardemusik statt.

Hundert Jahre nach der Gründung der Ingenieur-Akademie und nach dem siegreichen Ende der napoleonischen Kriege wurde mit der Unterzeichnung des Grundsteuerpatentes am 23. Dezember 1817 durch Kaiser Franz I. der Grundstein für die Einrichtung des nach ihm benannten Franziszeischen Katasters in Österreich und in den anderen Kronländern des Kaisertums Österreich gelegt (gekürzt und verändert nach www.kataster200.at). Basis dafür war die Vermessung aller Grundstücke des gesamten Staatsgebietes mit dem Messtisch. Diese Technik im Rahmen der Ingenieurvermessung war bereits von MARINONI wissenschaftlich, organisatorisch und technisch mit dem Mailänder Kataster perfektioniert worden.

Zwischen 1817 und 1861 wurden rund $300.000 \mathrm{~km}^{2}$ und mehr als 50 Millionen Grundstücke in ca. 30.000 Katastralgemeinden vermessen. Für jede Katastralgemeinde erfolgte eine eigene Bearbeitung. Die Grundstückgrenzen wurden in der sogenannten Urmappe auf ca. 165.000 Katastralmappen- 
blättern festgehalten. Diese Karten haben den Maßstab 1:2.880, damit entsprach 1 Zoll in der Mappe 40 Klaftern in der Natur. Als Folgemaßstäbe wurden im Hochgebirge 1:5.760 und in Stadtgebieten fallweise 1:1.440 verwendet. Die großmaßstäbigen Karten sind sehr detailreich und genau gezeichnet, beschriftet und koloriert. Die Ergebnisse der Vermessung und die Grundlage für die Bemessung der Grundsteuer waren (a) die Urmappe, (b) die Parzellenprotokolle, (c) die Grenzbeschreibungen sowie (d) die Steuerklassifizierungen. Es entstand derartig der erste umfassende moderne Liegenschaftskataster. Er verband eine Modernisierung der Grundsteuerbemessung (die amtliche Bewertung der Grundstückparzellen je nach Kulturgattung) mit der erstmals exakten Vermessung und kartographischen Darstellung der Grundstücke im gesamten Kaisertum Österreich. Die Kartenblattbereiche der Urmappe sind noch heute eine unschätzbare Quelle für topographische, siedlungsgeographische und naturwissenschaftliche Fragen. Die Urmappe gilt als gemeinsames Kulturgut aller Nachfolgestaaten Österreich-Ungarns und ist auch Kulturgut gemäß der Haager Konvention.

Die Führung des Katasters ist seit dem Evidenzhaltungsgesetz aus 1883 gesetzlich geregelt. Seit 1. Jänner 1969 ist das Vermessungsgesetz (BGB1. I Nr. 51/2016) die Rechtsgrundlage für die Anlage und Führung des Grenzkatasters. Die Grenzvermessungen und die Pläne der Vermessungsbefugten (Zivil- und Militärgeometer) sind damit die Grundlage für die Aktualisierung der Daten im Kataster. Seit 1985 sind sie zusammen mit den Daten des Grundbuchs in der Grundstücksdatenbank gespeichert und stehen öffentlich zur Verfügung. Seit 2004 ist auch die Katastralmappe als der graphische Teil des Katasters in Form der Digitalen Katastralmappe vorhanden. Die Aufgabe zur Führung des Katasters ist gesetzlich den Vermessungsämtern zugeordnet, die mit einem modernen Katasterführungssystem die Daten tagesaktuell halten.

Der Kataster in Österreich ist die Dokumentation der Lage der Grenzen von über 10 Millionen Grundstücken. Zusammen mit dem Grundbuch bildet er das Eigentumssicherungssystem in Österreich. Neben dem ursächlichen Zweck - für die Besteuerung - dient insbesondere der Grenzkataster auch der rechtlichen Sicherung der Grundstücksgrenzen. Folgeanwendungen sind z.B. solche im Grundstücksverkehr, in der Raumplanung (Flächenwidmungs- und Bebauungspläne der Gemeinden), in der Bodenschätzung (digitale Bodenschätzungsergebnisse) sowie im Agrarbereich (agrarische Operationen sowie Grundlage für Subventionen). Die Daten des Katasters sind öffentlich und online über das Portal www.bev.gv. at für Berechtigte verfügbar.

Anlässlich des Jubiläums „200 Jahre Kataster in Österreich“ wurden in den Jahren 2016 und 2017 folgende Veranstaltungen durchgeführt:

(a) Vom Bundesamt für Eich- und Vermessungswesen (BEV): 17.-19. Mai 2017 die 34. Fachtagung der Nachfolgestaaten der ehemaligen Monarchie, Wien (Tschechien, Slowakei, Ungarn, Kroatien, Slowenien, Trentino, Südtirol, Friaul-Julisch-Venetien und Österreich); 12. Juni 2017 Katasterfachtagung im Bundesamt für Eich- und Vermessungswesen; 1.-3. Oktober 2017 EuroGeographics-Generalversammlung, Wien; 4. Oktober 2017 Festveranstaltung im Festsaal der Österreichischen Akademie der Wissenschaften, Wien (vgl. BEV 2017); 5.-6. Oktober 2017 Common Vision Conference 2017 - Cadastre in Europe, Wien; 22. November 2017 Katastersymposium, Wien; November und Dezember 2017 Veranstaltungen in den Vermessungsämtern.

(b) Von der Kärntner Verwaltungsakademie: 6. Juli 2017 Veranstaltung Kataster und Grundbuch (www.verwaltungsakademie.ktn.gv.at/).

(c) Vom Niederösterreichischen Landesarchiv: 30. November 2016 Landesarchiv Sankt Pölten: Vermessen ...? Grenzen und Gerechtigkeit. 200 Jahre Franziszeischer Kataster; 16. Oktober 2017 die LAUT-Jahrestagung 2017 Weißenkirchen. MMag. Elisabeth LoINIG: 200 Jahre Österreichischer Kataster. Der Franziszeische Kataster (1817) - Von der Steuergerechtigkeit zur digitalen Kartennutzung.

(d) Vom Zwettler Stadtmuseum: 18. September 2017 Zwettler Museumsmontag. Thomas HaGMANN: 200 Jahre Kataster in Österreich. 
Bei diesen Veranstaltungen konnte öffentlichkeitswirksam (u.a. eigener TV-Film) die führende Rolle Österreichs im Bereich eines öffentlichen, flächendeckenden, genauen Mehrzweckkatasters und Grundbuchs vermittelt werden. Weiters wurde aufgezeigt, wie der Kataster auf der Grundlage von Tradition und Innovationen auf die Herausforderungen des digitalen Zeitalters reagiert hat. Faszinierend ist dabei die Dynamik, die in den vergangenen Jahrzehnten in verschiedene Weiterentwicklungen investiert wurde. Auch in den anderen Nachfolgestaaten der Monarchie besann man sich bald nach der politischen Wende auf dieses historische Kulturgut. Man ist auf gutem Weg, die neuen, sehr hohen technischen und rechtlichen Standards im Bereich des Katasterwesens zu erfüllen (BEV 2017, S. 335-399).

Unverständlich für eine Wertegemeinschaft, wie sich die Europäischen Union (EU) mit ihren zu Recht hohen rechtlichen und technischen Standards selbst definiert, ist die unterschiedliche Rechtssicherheit derzeit an Grund und Boden. In Südosteuropa und in weiten Teilen Südeuropas gibt es weder einen Grundstückkataster noch ein Grundbuch. Auch in allen anderen EU-Mitgliedstaaten gibt es höchst unterschiedliche Realisierungskonzepte. Es wird daher im Sinne der durch den Vertrag von Lissabon [Lisboa] vorgegebenen Rechtsstaatlichkeit der EU als vordringlich angesehen, hier endlich tätig zu werden. Gesprächs- und Fachforen gibt es zur Genüge. Was fehlt, ist der politische Wille, dieses sozioökonomische europäische Schlüsselprojekt auch umzusetzen. Natürlich sind große Widerstände vor allem von den Großgrundbesitzern und von der Orthodoxen Kirche (als größter Großgrundbesitzer z.B. in Griechenland) sowie von der zuständigen Beamtenschaft zu erwarten. Das ist der Grund für den langen Stillstand, zum Teil über Jahrhunderte. Ein neuer Anlauf (Geplant ist eine Initiative im Wege des Instituts für den Donauraum und Mitteleuropa 2018.) kann aber schlagartig die Akzeptanz der EU erhöhen, was durchaus im Sinne der überwiegenden Zahl der derzeitigen Verantwortungsträger und Meinungsbildner auf allen politischen Ebenen ist (FASCHING 2017a).

Historisches Vorbild für das angeregte europäische Großprojekt eines einheitlichen, harmonisierten Mehrzweckkatasters sowie entsprechender Basis- und topographischer Karten ist ein durchaus vergleichbares Projekt von Österreich-Ungarn nach der Besetzung von Bosnien-Herzegowina 1878. Die Schaffung eines Grundsteuerkatasters und die topographische und militärgeographische Landesaufnahme bildeten eine der wichtigen Grundlagen für die positive Entwicklung dieser rückständigen, ehemals osmanischen Provinz in kurzer Zeit in praktisch allen Lebensbereichen (FASCHING 2017a).

Die dritte (militär-)wissenschaftliche Großtat, mitten im Ersten Weltkrieg, mit Langzeitwirkung weltweit bis heute, war die Vereinbarung von Budapest 1917. Der Vertrag der damaligen Mittelmächte Deutschland, Österreich-Ungarn, sowie in weiterer Folge auch Bulgariens und des Osmanischen Reichs, war die Rechtsgrundlage zur Einführung des $3^{\circ}$-Gauß-Krüger-Abbildungssystems als geodätisches und geographisches Lagedatenbezugssystem.

Entwickelt wurden Gitternetze in (Militär-)Karten erstmalig von den Briten im Burenkrieg. Aber erst durch den massierten Artillerieeinsatz und durch das Planschießen im Ersten Weltkrieg gewannen Gitternetze im Bereich der Geodäsie und Kartographie an Bedeutung. Störend waren dabei die unterschiedlichen Kartenwerke und Suchgitter der Verbündeten. So wurde vor allem von preußischen Fachleuten das Gauß-Krüger-Abbildungssystem als zukunftsorientiertes einheitliches europäisches oder eurasisches Projekt entwickelt (MANG 1988). Dies, um einerseits die Hegemonie Preußens in Deutschland und in Europa zu stärken, anderseits um die Gunst der Stunde und die außenpolitischen Durchgriffsmöglichkeiten durch die Kriegsverhältnisse zu nutzen, innerhalb des fragilen Deutschen Kaiserreiches, der multiethnischen Doppelmonarchie, in den eroberten Gebieten im Osten sowie bei den Verbündeten in Südosteuropa und in Vorderasien - nach dem erhofften Siegfrieden der Mittelmächte - Fakten für die Nachkriegsordnung zu schaffen. In Friedenszeiten wäre so ein Monsterprojekt unter der Federführung Preußens - wenn überhaupt - nur mit einem sehr hohen Zeitaufwand möglich gewesen. Diese Tragweite wurde aber in Österreich nicht rich- 
tig erkannt, sehr wohl aber von Ungarn, das nur sehr zögerlich der Vereinbarung zustimmte. Wie die Geschichte gezeigt hat, hat sich das Gauß-Krüger-Abbildungssystem weltweit in Ost und West durchgesetzt, wenngleich in weiterentwickelten Formen, wie z.B. der Universalen Transversalen Mercator-Projektion (UTM).

Sehr interessant ist auch das damals vorgeschlagene Maßstabkonzept, das vom Verfasser als Anregung für Basis- und topographische Kartenwerke in Europa und weltweit übernommen wurde (FASCHING 2017a). Diese Maßstabreihe entspricht in allen Details unserem Euro-Währungssystem mit Maßstäben bzw. Münzen oder Banknoten bei den Basiskarten von 1:1.000, 1:2.000, 1:5.000, $1: 10.000$ und 1:20.000, bei den topographischen Karten 1:50.000, 1:100.000, 1:200.000 und 1:500.000 sowie bei den Weltkarten 1:1.000.000, 1:2.000.000 und 1:5.000.000.

Zusammenfassend kann festgestellt werden, dass viele tradierte Ansichten über die Entwicklung des Geoinformationswesens in Österreich und in Mitteleuropa einer Neubewertung bedürfen. Unumgänglich ist hierzu intensive Archivarbeit, die durch die modernen Informations- und Kommunikationstechniken erleichtert wird. Weiters ist ein interdisziplinärer Forschungsansatz eine Grundvoraussetzung, um zu halbwegs objektiven und gesicherten Ergebnissen zu gelangen. Verblüffende neue Ein- und Aussichten sollen Motivation sein, Traditionspflege und kulturelles Erbe nicht als bewahren der Asche, sondern als Ansporn für die Weitergabe des Feuers (Gustav MAHLER) zu verstehen.

\section{Literaturverzeichnis}

Bundesamt für Eich- und Vermessungswesen (BEV) (Hrsg.) (2017), Österreichisches Kulturgut. 200 Jahre Kataster. Wien, BEV.

FAsching G.L. (2017a), Das Projekt EuCart. Ein Konzept für neue kohärente Geoinformationen und Kartenwerke der Europäischen Union. In: Kartographische Nachrichten, 67, 1, S. 5-10.

FAsching G.L. (2017b), Wissenschaft, Technik, Produkte und Ausbildung auf dem Gebiet des Geoinformationswesens in Österreich 1717-2000. In: BUNDESMINISTERIUM FÜR LANDESVERTEIDIGUNG (Hrsg.), Führung - Technik - Wissenschaft. 300 Jahre militärtechnische Ausbildung in Österreich, S. 56-89. Graz, Vehling.

Hiermanseder M., KöNIG H. (2017), Johann Jakob von Marinoni - geadelt und getadelt, Schöpfer des Mailänder Katasters, Kartograph, Wissenschaftler. In: Österreichische Zeitschrift für Vermessung und Geoinformation, 105, 2, S. 60-141.

Kretschmer I., Dörflinger J., Wawrik F. (2004), Österreichische Kartographie. Von den Anfängen im 15. Jahrhundert bis zum 21. Jahrhundert (= Wiener Schriften zur Geographie und Kartographie, 15). Wien, Institut für Geographie und Regionalforschung, Kartographie und Geoinformation.

Lichtenberger E. (2009), Die Entwicklung der Geographie als Wissenschaft im Spiegel der Institutionspolitik und Biographieforschung. Vom Großstaat der k.u.k. Monarchie zum Kleinstaat der Zweiten Republik. In: Musil R., Staudacher Ch. (Hrsg.), Mensch, Raum, Umwelt, S. 13-51. Wien, Österreichische Geographische Gesellschaft.

Mang R. (1988), 70 Jahre Vereinbarung von Budapest. Ein Markstein für die Landesvermessung und Landesaufnahme in Österreich. Wien, Bundesministerium für Landesverteidigung.

Schels J.B. (1836), Oesterreichs Heer unter Kaiser Franz I. In: Oesterreichische militärische Zeitschrift, XXVIII, Erster Band, H. 1, Beitrag II, S. 37-95. 